\title{
Lack of Effect of Aciclovir on Metabolism of Theophylline and Expression of Hepatic Cytochrome P450 1 A2 in Rats
}

\author{
Masayuki Nadai, ${ }^{a}$ Miki Kato, ${ }^{b}$ Kazumasa Yasui, ${ }^{a}$ Masao Kimura,${ }^{b}$ Ying Lan Zhao, ${ }^{b, c}$ Jun Ueyama, ${ }^{d}$ \\ Yoshimi Tsunekawa, ${ }^{e}$ Hideo Yoshizumi, ${ }^{a}$ and Takaaki HasegawA ${ }^{*, b}$ \\ ${ }^{a}$ Department of Pharmaceutics, Faculty of Pharmacy, Meijo University; 150 Yagotoyama, Tenpaku, Nagoya 468-8503, \\ Japan: ${ }^{b}$ Department of Pharmacy and Pharmacokinetics, Aichi Medical University School of Medicine; 21 Nagakute-cho, \\ Aichi-gun, Aichi 480-1195, Japan: ${ }^{c}$ National Key Laboratory of Biotherapy, West China Hospital, Sichuan University; \\ Chengdu 610041, China: 'Department of Medical Technology, Nagoya University School of Health Sciences; \\ Daikominami, Higashi-ku, Nagoya 461-8673, Japan: and ${ }^{e}$ Department of Pharmaceutics, School of Pharmacy, Aichi \\ Gakuin University; 1-100 Kusumoto-cho, Chikusa-ku, Nagoya 462-8650, Japan. \\ Received October 18, 2006; accepted December 25, 2006; published online December 26, 2006
}

There is an interesting clinical report indicating that aciclovir, which is mainly excreted into urine, decreases the systemic clearance of theophylline by inhibiting cytochrome P450 (CYP) 1A2-mediated metabolism. In this study, we investigated the effect of aciclovir on the metabolism of theophylline, and on the activity and expression of hepatic CYP1A2 in rats. Theophylline $(10 \mathrm{mg} / \mathrm{kg})$ was injected intravenously into rats treated with two different dosages of aciclovir. When theophylline was simultaneously administered with aciclovir $(50 \mathrm{mg} / \mathrm{kg})$, the systemic clearance of theophylline and metabolic clearance of its major metabolites, 1-methyluric acid and 1,3-dimethyluric acid, were unchanged. In place of theophylline, when 1-methyl-3-propylxanthine $(2.5 \mathrm{mg} / \mathrm{kg})$, which is almost metabolized by CYP1A2 in rats, was coadministered intravenously with aciclovir $(50 \mathrm{mg} / \mathrm{kg})$, the pharmacokinetics of 1-methyl-3-propylxanthine was also unchanged. When theophylline was administered to rats pretreated with repeated intraperitoneal injections of aciclovir $(25 \mathrm{mg} / \mathrm{kg}$ twice daily for $3 \mathrm{~d})$, no significant differences in the systemic clearance of theophylline and its metabolic clearance to 1-methyluric acid and 1,3-dimethyluric acid were observed between the control and aciclovir-treated rats. This dosage of aciclovir did not change the activity of 7-ethoxyresorufin $O$-dealkylation, which is represented as CYP1A2 activity. In Western blot analysis, no significant change in the protein levels of hepatic CYP1A2 was observed between the control and aciclovirtreated rats. The present study suggests that aciclovir has no effect on the pharmacokinetics and metabolism of theophylline and on the activity and expression of hepatic CYP1A2 in rats.

Key words aciclovir; theophylline; drug interaction; metabolism; cytochrome P450 1A2 (CYP1A2)

Theophylline is widely used in the treatment of patients with obstructive airway diseases. It is well known that theophylline has a narrow therapeutic range being a plasma concentration of $10-20 \mu \mathrm{g} / \mathrm{ml}^{1,2)}$ and is extensively metabolized in humans. ${ }^{3,4)}$ Many articles have been published on the drug interactions of theophylline with other coadministered drugs mediated by cytochrome P450 (CYP) 1A2, which is the major metabolizing enzyme for theophylline. ${ }^{5-12)}$ For example, it is well known that histamine $\mathrm{H}_{2}$-antagonist cimetidine and quinolone antimicrobial agent enoxacin cause the risk of the development of serious side effects by inhibiting CYP1A2-mediated theophylline metabolism in the liver $7,9,13,14)$

The antivirus agent aciclovir, which has potent inhibitory activity against herpes simplex virus and varicella zoster virus ${ }^{15-17)}$ is known to be mainly eliminated from the kidney. ${ }^{18,19)}$ Drug interaction between aciclovir and concomitantly administered drugs, such as cimetidine, probenecid and mycophenolate that are primarily excreted into the urine has been reported. ${ }^{20-22)}$ However, there is an interesting clinical report indicating that total body clearance of theophylline is decreased by multiple oral administrations of aciclovir ( $800 \mathrm{mg}$ five times daily for two consecutive days) in healthy volunteers, ${ }^{23)}$ although theophylline is extensively metabolized in the liver. They proposed that the mechanism responsible for the decreases in theophylline clearance by coadministration of aciclovir might be due to inhibition of CYP1A2-mediated theophylline metabolism. However, the effect of aciclovir on the activity and expression of CYP1A2 remains unclear.

We have previously reported that rats are a useful animal model for predicting drug interaction between theophylline and other drugs in humans. ${ }^{24-29)}$ The aim of the present study was to investigate the effect of aciclovir on the pharmacokinetics and metabolism of theophylline, and on the activity and expression of hepatic CYP1A2 in rats. To further clarify whether aciclovir has an inhibitory effect against hepatic CYP1A2, we also investigated the effect of aciclovir on the pharmacokinetics of the xanthine derivative 1-methyl-3propylxanthine, which is almost completely metabolized by CYP1A2 in rats. ${ }^{29,30)}$

\section{MATERIALS AND METHODS}

Chemicals Theophylline, aciclovir, resorufin and 7ethoxyresorufin were purchased from Sigma Chemical Company (St. Louis, MO, U.S.A.). Aciclovir in the form of a commercial preparation for injection (Zovirax for IV infusion) was obtained from GlaxoSmithKline K.K. (Tokyo, Japan). Enprofylline, 1-methyl-3-propylxanthine and 1methyl-3-buthylxanthine were synthesized in our laboratory and were identical to those previously described. ${ }^{29,31)}$ Enprofylline and 1-methyl-3-buthylxanthine were used as internal standards for determination of concentrations of theophylline and 1-methyl-3-propylxanthine, respectively. Inulin was purchased from Nacalai Tesque (Kyoto, Japan). All other 
reagents are commercially available and were of analytical grade. All reagents were used without further purification. Theophylline, 1-methyl-3-propylxanthine and aciclovir were dissolved in saline.

Animals Eight-to-ten-week-old male Wistar rats (270 to $300 \mathrm{~g}$ ) were obtained from Japan SLC (Hamamatsu, Japan). The rats were housed under controlled environmental conditions (temperature of $24-25^{\circ} \mathrm{C}$ and humidity of $55 \pm 5 \%$ ) with a commercial food diet and water freely available to animals. All animal experiments were carried out in accordance with the guidelines in Faculty of Pharmacy, Meijo University and Aichi Medical University School of Medicine for the care and use of laboratory animals.

Pharmacokinetic Study One day before the start of the experiments, rats were anesthetized with an intraperitoneal administration of sodium pentobarbital $(25 \mathrm{mg} / \mathrm{kg}$ of body weight), and the right jugular vein was cannulated with polyethylene tubes for drug administration and blood collection. The rats received two different dosages of aciclovir; and control rats received an equivalent volume of saline in place of aciclovir. In the first study, the rats received simultaneously intravenous injection of theophylline $(10 \mathrm{mg} / \mathrm{kg})$ and aciclovir $(50 \mathrm{mg} / \mathrm{kg})$. In the second study, the rats received multiple intraperitoneal injections of aciclovir $(25 \mathrm{mg} / \mathrm{kg})$ twice daily (9:30 a.m. and 9:30 p.m.) for $3 \mathrm{~d}$ and on day 4 , theophylline $(10 \mathrm{mg} / \mathrm{kg})$ was injected intravenously $60 \mathrm{~min}$ after intraperitoneal injection of aciclovir $(25 \mathrm{mg} / \mathrm{kg})$ in the morning (9:30 a.m.). After intravenous injection of theophylline, rats were housed in metabolic cages, and urine was collected for $24 \mathrm{~h}$. Blood samples were collected at designated intervals of $5,10,20,30,45,60,90,120,180$ and $240 \mathrm{~min}$ after injection of theophylline.

To further investigate the effect of aciclovir on the metabolism of theophylline, we used a model compound, 1-methyl3-propylxanthine, which is mainly metabolized by CYP1A2 in the liver. ${ }^{29,30)}$ 1-Methyl-3-propylxanthine $(2.5 \mathrm{mg} / \mathrm{kg})$ was coadministered intravenously with aciclovir $(50 \mathrm{mg} / \mathrm{kg})$. Blood samples (about $0.2 \mathrm{ml}$ ) were collected at 5, 10, 20, 30, 45, 60, 90, 120 and 180 min after injection of drugs.

In a preliminary experiment, to measure the glomerular filtration rate (GFR) in rats pretreated with the multiple intraperitoneal injections of aciclovir, rats were cannulated with polyethylene tubes in the right jugular vein for drug administration, the left jugular artery for blood collection, the femoral vein for drug infusion, and the urinary bladder for urine collection. The rats received a bolus intravenous injection of inulin in a loading dose of $30 \mathrm{mg} / \mathrm{kg}$ followed by constant rate infusion (PHD 2000 infusion pump, Harvard, South Natick, Mass) of a 4\% mannitol solution delivering dose of $15 \mathrm{mg} / \mathrm{h}$ at a rate of $6 \mathrm{ml} / \mathrm{h}$ until the end of the experiment. After 60-min infusion, bile and urine were collected in preweighed tubes at 20-min intervals for $60 \mathrm{~min}$ throughout the experiment. Blood samples were taken at the midpoint of the bile and urine collection periods.

Plasma samples were immediately obtained by centrifugation of the blood samples at $4000 \times \boldsymbol{g}$ for $10 \mathrm{~min}$ at $4{ }^{\circ} \mathrm{C}$. The volume of urine samples was measured gravimetrically, with specific gravity assumed to be 1.0. Plasma and urine samples were stored at $-30{ }^{\circ} \mathrm{C}$ until analysis.

Hepatic Microsome Preparation for Enzyme Activity Assay and Western Blot Livers were obtained from rats receiving intraperitoneal injections of aciclovir $(25 \mathrm{mg} / \mathrm{kg})$ twice daily for $3 \mathrm{~d}$ and who were anesthetized with sodium pentobarbital $(25 \mathrm{mg} / \mathrm{kg}) 1 \mathrm{~h}$ after final administration of aciclovir or saline on the day of experiment. Then, the liver was excised after the perfusion of $150 \mathrm{ml}$ ice-cold saline to remove most of blood. The microsomes were prepared according to prior published methods ${ }^{32}$ ) with some modifications. Briefly, liver (approximately $3 \mathrm{~g}$ ) was homogenized at $4{ }^{\circ} \mathrm{C}$ with a Teflon homogenizer (10 strokes up and down) using a buffer solution consisting of $250 \mathrm{~mm}$ sucrose, $50 \mathrm{~mm}$ Tris$\mathrm{HCl}$ ( $\mathrm{pH} 7.6), 5 \mathrm{~mm} \mathrm{MgCl}_{2}$ and $10 \mathrm{~mm} \mathrm{KCl}$. The homogenate was centrifuged at $9000 \times \boldsymbol{g}$ for $25 \mathrm{~min}$, at $4{ }^{\circ} \mathrm{C}$. The supernatant was further centrifuged at $105000 \times \boldsymbol{g}$ for $60 \mathrm{~min}$ at $4{ }^{\circ} \mathrm{C}$ to obtain the microsomal fraction. The pellet obtained was resuspened in the buffer solution, and centrifuged at $105000 \times \boldsymbol{g}$ for $60 \mathrm{~min}$ at $4{ }^{\circ} \mathrm{C}$. The protein concentration of the microsomal fraction was measured by Bio-Rad Protein Assay (Bio-Rad Laboratories, Richmond, CA, U.S.A.) using bovine serum albumin (Sigma Chemical Company) as a standard. The microsomal fraction was kept at $-80{ }^{\circ} \mathrm{C}$ until analysis of enzyme activity and Western blot.

7-Ethoxyresorufin Dealkylation Activity The activity of CYP1A2 in hepatic microsomes was assessed as the formation of resorufin from 7-ethoxyresorufin based on the method reported previously. ${ }^{33)}$ Briefly, a volume of $1.7 \mathrm{ml}$ of $50 \mathrm{~mm}$ phosphate-buffered incubation medium $(\mathrm{pH} 7.4)$ containing $3.3 \mathrm{~mm} \mathrm{MgCl}_{2}, 1.3 \mathrm{~mm} \beta$-NADP ${ }^{+}, 3.3 \mathrm{~mm}$ glucose6-phosphate, $0.4 \mathrm{U} / \mathrm{ml}$ glucose-6-phosphate dehydrogenase (final concentration of reaction mixture, respectively) and $200 \mu \mathrm{l}$ of the obtained microsomal protein (about $3.0 \mathrm{mg}$ protein $/ \mathrm{ml}$ ) was placed in a sample tube and preincubated for $1.5 \mathrm{~min}$ at $37^{\circ} \mathrm{C}$. The reaction was initiated by addition of $100 \mu \mathrm{l}$ of 7-ethoxyresorufin $(100 \mu \mathrm{M})$ as a substrate. After incubation for $0.5,1$ or $3 \mathrm{~min}$ at $37^{\circ} \mathrm{C}$, the reaction was terminated by adding $4 \mathrm{ml}$ of ice-cold acetonitrile. The samples were vortexed for $30 \mathrm{~s}$ and were centrifuged at $11000 \times \boldsymbol{g}$ for $10 \mathrm{~min}$. The supernatant was used for measurement with a spectrofluorophotometer (FP-777, JASCO, Japan) set at 550 $\mathrm{nm}$ (excitation) and $586 \mathrm{~nm}$ (emission). Standard curves for resorufin $(20$ to $600 \mathrm{ng} / \mathrm{ml})$ were shown to be linear.

Western Blot Analysis for CYP1A2 Western blot analysis for CYP1A2 was performed according to the methods described previously. ${ }^{34-36)}$ Briefly, the microsomal protein $(1 \mu \mathrm{g})$ was separated by electrophoresis on $10 \%$ polyacrylamide gel containing $0.1 \%$ sodium dodecyl sulfate (SDS) and transferred to a polyvinylidene difluoride (PVDF) membrane (Millipore Company, Bedford, MA, U.S.A.). The membrane was blocked in phosphate-buffered saline (PBS) containing $0.1 \%$ Tween 20 and $4 \%$ nonfat dry milk, and detected by goat polyclonal antibody to rat CYP1A2 (Daiichi Pure Chemicals). Immune complexes were visualized using horseradish peroxidase-labeled secondary antibody, anti-goat IgG (Sigma Chemicals), with ECL Western blot detection reagents (Amersham Biosciences, Piscataway, NJ, U.S.A.).

To quantify the relative levels of CYP1A2 in each gel, the intensity of the stained bands was measured by the NIH image program (Bethesda, MD, U.S.A.). The levels were expressed as $100 \%$ of that of the control rats.

Drug Analysis Concentrations of theophylline in plasma and urine, and its metabolites (1-methyluric acid and 1,3-dimethyluric acid) in urine were determined by HPLC. The ap- 
paratus was Shimadzu LC-10A system (Kyoto, Japan) equipped with a UV spectrophotometric detector (SPD-10 AV) consisting of an LC-10A liquid pump and an SIL-6A autoinjector. The conditions and parameters for determination of plasma theophylline concentration were according to the method described previously with some modification. ${ }^{37}$ Briefly, $350 \mu \mathrm{l}$ of methanol containing enprofylline $(0.5$ $\mu \mathrm{g} / \mathrm{ml})$ as an internal standard was added to $50 \mu \mathrm{l}$ of plasma sample and mixed. After centrifugation at $6000 \times \boldsymbol{g}$ for 10 min, $300 \mu \mathrm{l}$ of supernatant was evaporated to dryness under an $\mathrm{N}_{2}$ gas stream at $50^{\circ} \mathrm{C}$. The residue obtained was dissolved in $250 \mu \mathrm{l}$ of the mobile phase, and injected to HPLC $(150 \mu \mathrm{l})$. The UV detector was set at $274 \mathrm{~nm}$, and the column (Cosmosil 5C18-MS-II $4.6 \mathrm{~mm}$ i.d. $\times 150 \mathrm{~mm}$, Nacalai Tesque, Kyoto, Japan) was heated at $40{ }^{\circ} \mathrm{C}$. The mobile phase was a mixture of $30 \mathrm{~mm} \mathrm{KH}_{2} \mathrm{PO}_{4}$ buffer $(\mathrm{pH} 4.0)$ and methanol $(88: 12[\mathrm{vol} / \mathrm{vol}])$, and the flow rate was 1.2 $\mathrm{ml} / \mathrm{min}$.

For analysis of theophylline, 1-methyluric acid and 1,3-dimethyluric acid in urine, diluted urine samples were passed through a membrane filter with a pore size of $0.45 \mu \mathrm{m}$ and were injected directly onto the HPLC. The conditions and parameters were according to the method described previously. ${ }^{29)}$ The mobile phase consisted of $10 \mathrm{mM} \mathrm{KH}_{2} \mathrm{PO}_{4}$ buffer ( $\mathrm{pH} 2.5$ ) and methanol (96:4 [vol/vol]), and the flow rate was $1.5 \mathrm{ml} / \mathrm{min}$. The UV detector was set at $274 \mathrm{~nm}$, and the column was heated to $40^{\circ} \mathrm{C}$. For calculation, standard curves for theophylline and its metabolites, 1-methyluric acid and 1,3-dimethyluric acid proved to be linear for concentrations ranging from 0.25 to $25 \mu \mathrm{g} / \mathrm{ml}$.

Concentrations of 1-methyl-3-propylxanthine in plasma were measured by HPLC based on the method reported previously. ${ }^{37)}$ Briefly, $250 \mu$ of methanol containing 1-methyl-3buthylxanthine $(0.4 \mu \mathrm{g} / \mathrm{ml})$ as an internal standard was added to $50 \mu \mathrm{l}$ of plasma sample and mixed. After centrifugation at $6000 \times \mathbf{g}$ for $10 \mathrm{~min}$, the resulting supernatant $(200 \mu \mathrm{l})$ was evaporated to dryness under a nitrogen gas stream at $50{ }^{\circ} \mathrm{C}$. The residue was reconstituted with $200 \mu \mathrm{l}$ of the mobile phase and injected into the HPLC. The UV detector was set at $274 \mathrm{~nm}$, and the column temperature was $35^{\circ} \mathrm{C}$. The mobile phase was a mixture of $30 \mathrm{mM} \mathrm{KH}_{2} \mathrm{PO}_{4}$ buffer $(\mathrm{pH} 3.0)$ and methanol $(60: 40[\mathrm{vol} / \mathrm{vol}])$, and the flow rate was 1.2 $\mathrm{ml} / \mathrm{min}$. The within-day and between-day coefficients of variation for these assays were less than $6 \%$. No interference with the peaks of theophylline and its metabolites, and 1methyl-3-propylxanthine was observed in any samples.

Inulin concentrations in plasma and urine were determined spectrophotometrically by the methods of Dische and Borenfreund. ${ }^{38)}$

Data Analysis Concentration-time data for theophylline and 1-methyl-3-propylxanthine in each rat were analyzed individually using a noncompartmental model. The area under the curve $(A U C)$ was calculated by the trapezoidal rule for values up to the last measured concentration in plasma and was extrapolated to infinity by adding the following: the value of the last measured concentration in plasma divided by the terminal elimination rate constant, which was calculated by determining the slope of the least-squares regression line from the terminal portion of the log concentration-time data. Systemic clearance $\left(C L_{\mathrm{SYS}}\right)$ was calculated as $C L_{\mathrm{SYS}}=$ dose/AUC. The mean residence time (MRT) was calculated as $M R T=A U M C / A U C$, where $A U M C$ represents the area under the first moment of the curve. The steady-state volume of distribution $\left(V_{\mathrm{SS}}\right)$ was calculated as $V_{\mathrm{SS}}=C L_{\mathrm{SYS}} \times M R T$. The renal clearance $\left(C L_{\mathrm{R}}\right)$ was calculated as $C L_{\mathrm{R}}=C L_{\mathrm{SYS}} \times f_{\mathrm{e}}$, where $f_{\mathrm{e}}$ represents the fraction of urinary excretion of unchanged theophylline. Metabolic clearance $\left(C L_{\mathrm{M}}\right)$ of theophylline to each metabolite was calculated as $C L_{\mathrm{M}}=$ $f_{\mathrm{M}} \times C L_{\mathrm{SYS}}$, where $f_{\mathrm{M}}$ represents the recovery of each metabolite in urine as a fraction of the dose administered.

Statistical Analysis All data are expressed as means S.D. Statistical differences between the control and aciclovirtreated rats were assessed by Student's $t$-test, and $p$ values less than 0.05 were taken as significant.

\section{RESULTS}

Mean semilogarithmic plots of plasma concentration-time data for theophylline after a single intravenous administration at a dose of $10 \mathrm{mg} / \mathrm{kg}$ in control rats and rats treated with a single intravenous injection or intraperitoneally repetitive injections of aciclovir are illustrated in Fig. 1. As shown in Fig. 1, either a single intravenous injection or multiple intraperitoneal injections of aciclovir did not change the plasma concentration profiles of theophylline. Corresponding pharmacokinetic parameters of theophylline are summarized in Table 1. No significant differences in the pharmacokinetic parameters of systemic clearance $\left(C L_{\mathrm{SYS}}\right)$, volume of distri-
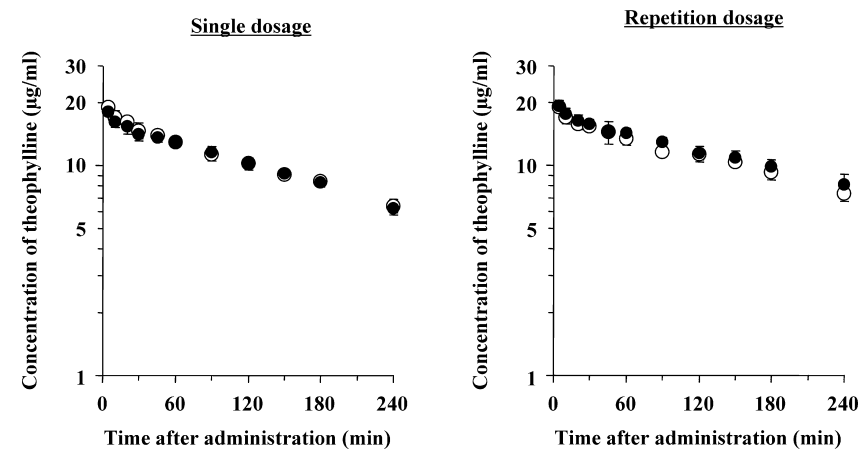

Fig. 1. Mean Plasma Concentration-Time Data for Theophylline after Intravenous Administration in Rats Treated with a Single Intravenous Injection (Left) and Multiple Intraperitoneal Injections (Right) of Aciclovir

Each symbol represents the mean \pm S.D. $(n=5-6)$. See Materials and Methods for details and drug administration. No significant difference was observed between control and aciclovir-treated rats. When the standard error is small, it is included in the symbol. Symbols: $\bigcirc$, control rats; $\bigcirc$, aciclovir-treated rats.

Table 1. Effect of Single Intravenous Injection and Multiple Intraperitoneal Injections of Aciclovir on Pharmacokinetic Parameters of Theophylline after Intravenous Administration in Rats

\begin{tabular}{cccc}
\hline \hline Treatment & $\begin{array}{c}C L_{\mathrm{SYS}} \\
(\mathrm{l} / \mathrm{h} / \mathrm{kg})\end{array}$ & $\begin{array}{c}V_{\mathrm{SS}} \\
(\mathrm{l} / \mathrm{kg})\end{array}$ & $\begin{array}{c}M R T \\
(\mathrm{~h})\end{array}$ \\
\hline $\begin{array}{c}\text { Single dosage } \\
\text { Control }\end{array}$ & $0.145 \pm 0.009$ & $0.588 \pm 0.038$ & $4.09 \pm 0.45$ \\
Aciclovir & $0.149 \pm 0.011$ & $0.583 \pm 0.046$ & $3.95 \pm 0.58$ \\
Repetition dosage & & & \\
Control & $0.129 \pm 0.011$ & $0.567 \pm 0.019$ & $4.43 \pm 0.46$ \\
Aciclovir & $0.113 \pm 0.013$ & $0.570 \pm 0.036$ & $5.10 \pm 0.57$ \\
\end{tabular}

Each value is represented as mean \pm S.D. $(n=5-6) . C L_{\mathrm{SYS}}, V_{\mathrm{SS}}$ and $M R T$ represent systemic clearance, volume of distribution at steady state and mean residence time, respectively. No significant differences in all parameters were observed between control and aciclovir-treated rats. 
Table 2. Effect of Single Intravenous Injection and Multiple Intraperitoneal Injections of Aciclovir on Urinary Recovery of Theophylline and Its Metabolites after Intravenous Administration in Rats

\begin{tabular}{|c|c|c|c|}
\hline \multirow{3}{*}{ Treatment } & \multicolumn{3}{|c|}{$\%$ total recovery in urine } \\
\hline & \multicolumn{2}{|c|}{ Metabolite } & \multirow{2}{*}{ Theophylline } \\
\hline & $\begin{array}{l}\text { 1-Methyluric } \\
\text { acid }\end{array}$ & $\begin{array}{l}\text { 1,3-Dimethyluric } \\
\text { acid }\end{array}$ & \\
\hline \multicolumn{4}{|l|}{ Single dosage } \\
\hline Control & $35.4 \pm 5.4$ & $35.1 \pm 4.5$ & $18.2 \pm 1.8$ \\
\hline Aciclovir & $35.0 \pm 4.2$ & $36.3 \pm 4.4$ & $14.5 \pm 4.6$ \\
\hline \multicolumn{4}{|c|}{ Repetition dosage } \\
\hline Control & $38.0 \pm 2.6$ & $36.4 \pm 3.1$ & $20.2 \pm 2.6$ \\
\hline Aciclovir & $42.1 \pm 3.1^{a)}$ & $41.2 \pm 2.2^{a)}$ & $13.7 \pm 3.7^{a)}$ \\
\hline
\end{tabular}

Each value is represented as mean \pm S.D. $(n=5-6)$. Values of metabolites are ex pressed on a molar basis and represent the amount excreted in $24 \mathrm{~h}$ as percentage of theophylline dose. a) Significantly different from control $(p<0.05)$.
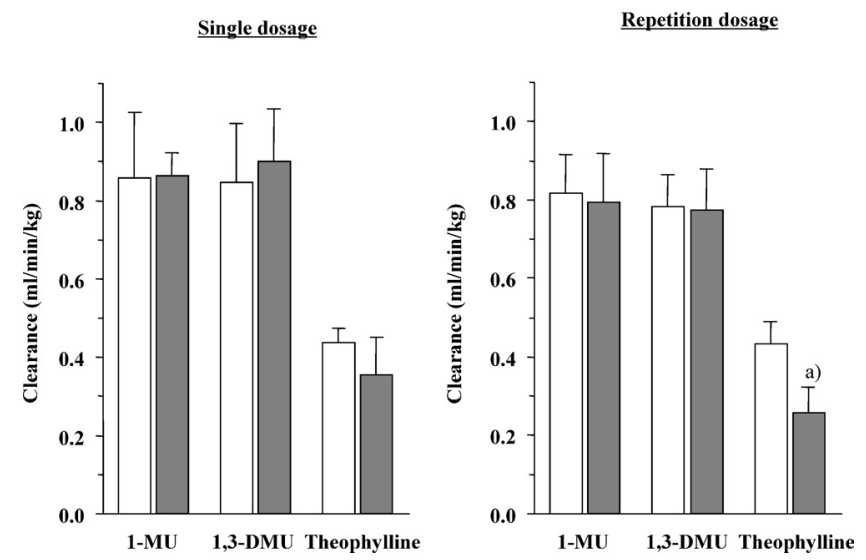

Fig. 2. Metabolic and Renal Clearance of Theophylline after Intravenous Administration in Rats Treated with a Single Intravenous Injection (Left) and Multiple Intraperitoneal Injections (Right) of Aciclovir

1-MU, 1-methyluric acid; DMU, 1,3-dimethyluric acid. Each column represents the mean \pm S.D. $(n=5-6)$. See Materials and Methods for details and drug administration. "a" indicates values significantly different from the control values $(p<0.05)$. Columns: $\square$, control rats; $\boldsymbol{\square}$, aciclovir-treated rats.

bution at steady state $\left(V_{\mathrm{SS}}\right)$ and mean residence time $(M R T)$ for theophylline were observed between the control and aciclovir-treated rats. Table 2 indicates the urinary recovery of theophylline and its metabolites during $24 \mathrm{~h}$ after intravenous injection of theophylline in the control and aciclovir-treated rats. The urinary recovery of theophylline and its metabolites was not changed by a single intravenous injection of aciclovir. However, the multiple intraperitoneal injections of aciclovir significantly decreased urinary recovery of theophylline and increased the two metabolites. As shown in Fig. 2 , neither the single intravenous injection nor multiple intraperitoneal injections of aciclovir altered the metabolic clearance of theophylline to 1-methyluric acid and 1,3-dimethyluric acid. However, multiple intraperitoneal injections of aciclovir significantly decreased renal clearance $\left(C L_{R}\right)$ of theophylline.

Aciclovir is known to induce acute renal failure in humans. Initially, to investigate the effect of the multiple intraperitoneal injections of aciclovir on kidney function, we measured inulin clearance, which represents the glomerular filtration rate (GFR). No significant difference in the

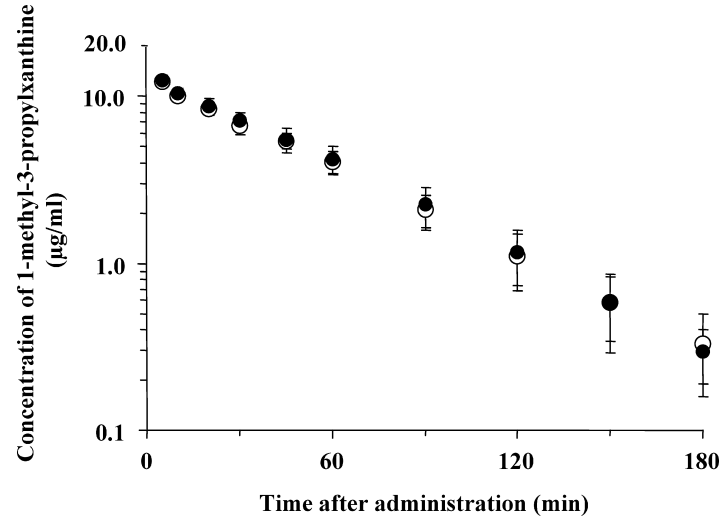

Fig. 3. Mean Plasma Concentration-Time Data for 1-Methyl-3-propylxanthine after Intravenous Injection of Aciclovir

Each symbol represents the mean \pm S.D. $(n=4-6)$. See Materials and Methods for details and drug administration. No significant difference was observed between contro and aciclovir-treated rats. When the standard error is small, it is included in the symbol. Symbols: $\bigcirc$, control rats; 9 , aciclovir-treated rats.

Table 3. Effect of Single Intravenous Injection of Aciclovir on Pharmacokinetic Parameters of 1-Methyl-3-propylxanthine in Rats

\begin{tabular}{lcccc}
\hline \hline Treatment & $\begin{array}{c}C L_{\mathrm{SYS}} \\
(\mathrm{l} / \mathrm{h} / \mathrm{kg})\end{array}$ & $\begin{array}{c}V_{\mathrm{SS}} \\
(1 / \mathrm{kg})\end{array}$ & $\begin{array}{c}M R T \\
(\mathrm{~h})\end{array}$ & $\begin{array}{c}t_{1 / 2} \\
(\mathrm{~h})\end{array}$ \\
\hline Control & $0.235 \pm 0.030$ & $0.186 \pm 0.012$ & $0.801 \pm 0.109$ & $0.572 \pm 0.078$ \\
Aciclovir & $0.228 \pm 0.035$ & $0.178 \pm 0.015$ & $0.787 \pm 0.089$ & $0.543 \pm 0.048$ \\
\hline
\end{tabular}

Each value is represented as mean \pm S.D. $(n=4-6)$. $C L_{\mathrm{SYS}}, V_{\mathrm{SS}}, M R T$ and $t_{1 / 2}$ represent systemic clearance, volume of distribution at steady state, mean residence time and terminal half-life, respectively. No significant differences in all parameters were observed between control and aciclovir-treated rats.

glomerular filtration rate was observed between the control $(7.29 \pm 0.27 \mathrm{ml} / \mathrm{min} / \mathrm{kg})$ and aciclovir-treated $(6.62 \pm 0.81$ $\mathrm{ml} / \mathrm{min} / \mathrm{kg}$ ) rats, indicating that aciclovir has no effect on kidney function.

The effect of simultaneous intravenous injection of aciclovir on the pharmacokinetics of 1-methyl-3-propylxanthine was investigated. Figure 3 shows the plasma concentrationtime profile of 1-methyl-3-propylxanthine in untreated and aciclovir-treated rats. Aciclovir did not change the disappearance of 1-methyl-3-propylxanthine from plasma. No significant differences in the plasma concentration profiles and pharmacokinetic parameters of 1-methyl-3-propylxanthine were observed between control and aciclovir-treated rats (Table 3).

To clarify the effect of the multiple intraperitoneal injection of aciclovir on the activity and expression of hepatic CYP1A2, we measured the activity of 7-ethoxyresorufin $O$ deethylation and the protein levels of hepatic CYP1A2 by using Western blot analysis. As shown in Fig. 4, aciclovir did not change either the activity or expression of CYP1A2 in the liver.

\section{DISCUSSION}

It has been reported that aciclovir is primarily excreted into the urine by glomerular filtration and tubular secretion in unchanged form, and a small amount of the administered dose $(8-14 \%)$ is recovered as the metabolite 9-carboxymethoxymethylguanine in patients with normal kidney function. ${ }^{19)}$ In mice and rats, approximately $95 \%$ of aciclovir 

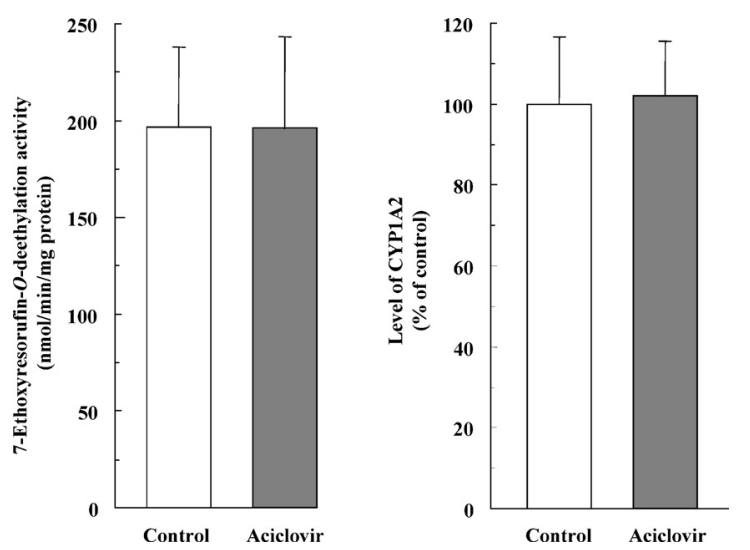

Fig. 4. 7-Ethoxyresorufin $O$-Deethylation Activity (Left) and Expression of Hepatic CYP1A2 (Right) in Rats Treated with Multiple Intraperitoneal Injections of Aciclovir

Data of Western blot analysis are expressed as $100 \%$ of the mean value of the control rats. Each column represents the mean \pm S.D. $(n=4)$. Columns: $\square$, control rats; $\mathbf{\square}$, aciclovir-treated rats.

administered is also recovered in the urine as an unchanged form. ${ }^{18)}$ In spite of the fact that most of the administered aciclovir is not metabolized in animals and humans, the inhibitory effect of aciclovir against theophylline metabolism has been reported by Maeda et al. ${ }^{23)}$ who reported that total body clearance of theophylline and the formation clearance of its major metabolites, 1-methyluric acid, 3-methylxanthine and 1,3-dimethyluric acid from theophylline, decreased in healthy male volunteers who received oral aciclovir $800 \mathrm{mg}$ five times daily for $2 \mathrm{~d}$. They proposed that this mechanism is due to inhibition of CYP1A2-mediated theophylline metabolism by aciclovir. Therefore, there is a possibility that aciclovir modifies the pharmacokinetics of several drugs, including caffeine, ${ }^{39,40)}$ phenacetin ${ }^{39,41)}$ clozapine $^{42)}$ and propranolol, ${ }^{43)}$ which are typical substrates for CYP1A2, and induces side effects. However, to our knowledge, there is no information available whether aciclovir can modify the activity and expression of hepatic CYP1A2. Then, we examined the effect of aciclovir on the activity and expression of CYP1A2 and the precise mechanism of drug interaction between theophylline and aciclovir in rats.

The bioavailability of aciclovir after oral administration is reported to be approximately $20 \%$ in healthy volunteers and in patients with malignant hematological disease. ${ }^{19,44)}$ Fujioka $e t \mathrm{al}^{45)}$ reported that the absolute bioavailability of aciclovir after oral administration of $20 \mathrm{mg} / \mathrm{kg}$ in Wistar rats was $7.3 \%$. However, a study on the pharmacokinetics of aciclovir in subjects with end-stage renal disease showed that the bioavailability of aciclovir after intraperitoneal administration in patients receiving continuous ambulatory peritoneal dialysis was approximately $60 \%$, suggesting that the peritoneal cavity is an alternative route for aciclovir administration in patients with poor vascular access. ${ }^{46)}$ In addition, a part of aciclovir administered by intraperitoneal injection is absorbed via mesenteric vessels and flowed in the portal vein as well as oral administration. In the present study, we chose intravenous and intraperitoneal injections as a dosing route of aciclovir, and the dose was chosen on the basis of clinical studies reported by Maeda and colleagues. ${ }^{23)}$ Then, to clarify the mechanism of drug interaction between theophylline and aciclovir, we examined the effect of aciclovir on the pharma- cokinetics and metabolism of theophylline by a single intravenous injection of aciclovir $(50 \mathrm{mg} / \mathrm{kg})$ and multiple intraperitoneal injections of aciclovir $(25 \mathrm{mg} / \mathrm{kg}$ twice a day for $3 \mathrm{~d}$ ). The values of the pharmacokinetic parameters and urinary recovery of theophylline and its metabolites observed in control rats were comparable to those of our previous studies. ${ }^{12,29)}$ Unexpectedly, results obtained from the present study showed that neither single intravenous injection nor multiple intraperitoneal injections of aciclovir altered the plasma concentration-time profiles and pharmacokinetic parameters of theophylline. In addition, aciclovir had no effect on the urinary recovery of theophylline metabolites, 1methyluric acid and 1,3-dimethyluric acid, during a urine collection period of $24 \mathrm{~h}$, which is about 7 -fold longer than the plasma half-life of theophylline (approximately $3 \mathrm{~h}$ ), although slight increases in the urinary recovery of the two metabolites were observed in rats treated with multiple intraperitoneal injections of aciclovir. Similarly, no significant changes in the metabolic clearance of theophylline to the two metabolites were observed between the control and aciclovirtreated rats. From these pharmacokinetic findings, it was suggested that aciclovir has no effect on CYP1A2-mediated metabolism of theophylline in rats.

We previously reported that the xanthine derivative 1methyl-3-propylxanthine is almost completely metabolized in rats, ${ }^{30)}$ and the systemic clearance of 1-methyl-3-propylxanthine was significantly decreased by approximately $80 \%$ after pretreatment with the quinolone antimicrobial agent enoxacin, a typical CYP1A2 inhibitor, indicating that 1methyl-3-propylxanthine in rats might be mainly metabolized by CYP1A2 as well as theophylline in humans. ${ }^{26)}$ Further, the present study used 1-methyl-3-propylxanthine as a model drug of theophylline and examined the effect of aciclovir on the pharmacokinetics of 1-methyl-3-propylxanthine. As can be seen in theophylline, no significant changes in the plasma concentration-time profile and pharmacokinetic parameters of 1-methyl-3-propylxanthine were observed between the control and aciclovir-treated rats. These results suggest at least that aciclovir has no effect on CYP1A2-mediated metabolism.

Finally, we investigated whether aciclovir modifies the drug metabolizing enzyme activity of CYP1A2 by measuring the activity of 7-ethoxyresorufin $O$-deethylation in the liver. As shown in Fig. 4, no significant change in the formation of resorufin from 7-ethoxyresorufin was found in hepatic microsomes prepared from the control and aciclovir-treated rats, indicating that aciclovir did not affect the activity of CYP1A2. In addition, results of Western blot analysis showed that the aciclovir did not alter the protein levels of hepatic CYP1A2, which agreed well with the results of $7-$ ethoxyresorufin $O$-deethylation activity. These findings supported results that aciclovir did not affect the pharmacokinetics of theophylline and 1-methyl-3-propylxanthine in vivo.

Unexpectedly, the results of the present study using rats could not show the inhibitory effect of aciclovir against the metabolism of both theophylline and 1-methyl-3-propylxanthine, which did not support a clinical report indicating that aciclovir decreased the total body clearance of theophylline and the formation clearance of theophylline metabolites in healthy volunteers. ${ }^{23)}$ The discrepancy between results in rats and humans might be explained by differential selectivity 
and an affinity of aciclovir for CYP1A2 between rats and humans. For example, Eagling et al. ${ }^{47)}$ reported that the inhibitory potency of furafylline, which is a potent selective inhibitor of CYP1A2 in humans against phenacetin $O$-deethylation, is 20-fold stronger in human liver microsomes $\left(\mathrm{IC}_{50}=0.48 \mu \mathrm{M}\right)$ than that in rat liver microsomes $\left(\mathrm{IC}_{50}=20.8\right.$ $\mu \mathrm{M})$, which might be due to differences in the structure of CYP1A2 protein between rats and humans. They also pronounced that some inhibitors for drug metabolizing enzymes such as furafylline, sulphaphenazole and ketoconazole, potent selective inhibitors for human CYP1A2, CYP2C9 and CYP3A4, do not exhibit the same selectivity in microsomes of human and rat livers. Kobayashi and colleagues ${ }^{48)}$ have reported similar findings that chemical inhibitor probes for human P450 isoforms do not show the same selectivity for the corresponding rat CYP isoforms. However, our previous experiments using rats demonstrated that enoxacin significantly decreased the systemic clearance of theophylline by inhibiting hepatic metabolism and that telithromycin significantly inhibited the theophylline metabolism by decreasing the activity and expression of hepatic CYP1A2. ${ }^{129)}$ Based on these results, the present study, at least, suggests that the inhibitory effect of aciclovir against CYP1A2 is weaker than these drugs in rats.

It is known that the systemic clearance of theophylline in humans corresponds mostly to the metabolic clearance, whereas the contribution of renal clearance of theophylline to the systemic clearance in rats is larger than that in humans. In the present study, significant decrease in the renal clearance of theophylline was observed in rats pretreated with the multiple intraperitoneal injections of aciclovir. Considering that aciclovir did not decrease the glomerular filtration rate (GFR), it is unlikely that the decreased renal clearance of theophylline by aciclovir was caused by renal dysfunction. Kuh and Shim ${ }^{49)}$ reported that theophylline is excreted into urine by glomerular filtration, tubular secretion and tubular reabsorption in rats. It is possible that aciclovir may affect the renal excretion mechanism of theophylline, although the precise mechanism of the decreased renal clearance of theophylline by aciclovir observed in this study is not clear.

In conclusion, the present study reports that aciclovir has no significant effect on the pharmacokinetics and metabolism of theophylline in rats, which can not support metabolic drug interaction between theophylline and concomitantly administered aciclovir observed in humans. Further studies are needed to elucidate the mechanism by which aciclovir inhibits CYP1A2-mediated theophylline metabolism in humans.

Acknowledgement This work was supported in part by a Grant-in-Aid for Scientific Research (17590500) from the Ministry of Education, Culture, Sports, Science and Technology of Japan.

\section{REFERENCES}

1) Levy G., Koysooko R., J. Pediatr., 86, 789-793 (1975).

2) Hendeles L., Bighley L., Richardson R. H., Hepler C., Carmichael J., Drug Int. Clin. Pharm., 11, 12-18 (1977).

3) Jenne J. W., Nagasawa H. T., Thompson R. D., Clin. Pharmacol. Ther, 19, 375-381 (1976)

4) Campbell M. E., Grsnt D. M., Inaba T., Kalow W., Drug Metab. Dis- pos., 15, 237-249 (1987)

5) Upton R. A., Clin. Pharmacokinet., 20, 66-80 (1991).

6) Upton R. A., Clin. Pharmacokinet., 20, 135-150 (1991).

7) Von Rosenstiel N., Adam D., Drugs, 47, 872—901 (1994).

8) Perucca E., Gatti G., Spina E., Clin. Pharmacokinet., 27, 175-190 (1994).

9) Negro R. D., Clin. Pharmacokinet., 35, 135-150 (1998)

10) Rodvold K. A., Clin. Pharmacokinet., 37, 385-398 (1999).

11) Ohnishi A., Method Find. Exp. Clin. Pharmacol., 22, 253-258 (2000).

12) Nosaka H., Nadai M., Kato M., Yasui K., Yoshizumi H., Miyoshi M., Zhao Y. L., Baba K., Takagi K., Hasegawa T., Life Sci., 79, 50-56 (2006).

13) Jackson J. E., Powell J. R., Wandell M., Bentley J., Dorr R., Am. Rev. Respir. Dis., 123, 615-617 (1981).

14) Wijnands W. J. A., Vree T. B., van Herwaarden C. L. A., Br. J. Clin. Pharmacol., 20, 583-588 (1985).

15) Schaeffer H. J., Beauchamp L., de Miranda P., Elion G. B., Bauer D. J., Collins P., Nature (London), 272, 583-585 (1978).

16) Collins P., Bauer D. J., J. Antimicrob. Chemother, 5, 431-436 (1979).

17) Biron K. K., Elion G. B., Antimicrob. Agents Chemother, 18, 443447 (1980).

18) De Miranda P., Krasny H. C., Page D. A., Elion G. B., J. Pharmacol. Exp. Ther., 219, 309-315 (1981).

19) De Miranda P., Blu M. R., J. Antimicrob. Chemother., 12, 29-37 (1983).

20) De Bony F., Tod M., Bidault R., On N. T., Posner J., Rolan P., Antimicrob. Agents Chemother, 46, 458-463 (2002).

21) Gimenez F., Foeillet E., Bourdon O., Weller S., Garret C., Bidault R., Singlas E., Clin. Pharmacokinet., 43, 685-692 (2004).

22) Gwak H. S., Oh J. H., Han H. K., J. Pharm. Pharmacol., 57, 393-398 (2005).

23) Maeda Y., Konishi T., Omoda K., Takeda Y., Fukuhara S., Fukuzawa M., Ohune T., Tsuya T., Tsukiai S., Biol. Pharm. Bull., 19, 15911595 (1996).

24) Takagi K., Hasegawa T., Ogura Y., Suzuki R., Yamaki K., Watanabe T., Kitazawa S., Satake T., J. Asthma, 25, 63-71 (1988).

25) Kuzuya T., Takagi K., Apichartpichean R., Muraoka I., Nadai M., Hasegawa T., J. Pharmacobio-Dyn., 12, 405-409 (1989).

26) Hasegawa T., Nadai M., Kuzuya T., Apichartpichean R., Takagi K., Miyamoto K., J. Pharm. Pharmacol., 42, 767-772 (1990).

27) Hasegawa T., Kuzuya T., Apichartpichean R., Nadai M., Nitta A., Takagi K., Nabeshima T., Pharmacol. Toxicol., 69, 5-8 (1991).

28) Hasegawa T., Nadai M., Haghgoo S., Yamaki K., Takagi K., Nabeshima T., Antimicrob. Agents Chemother., 39, 2138-2140 (1995).

29) Nadai M., Hasegawa T., Kuzuya T., Muraoka I., Takagi K., Yoshizumi H., Antimicrob. Agents Chemother, 34, 1739-1743 (1990).

30) Apichartpichean R., Hasegawa T., Nadai M., Kuzuya T., Nabeshima T., J. Pharm. Pharmacol., 43, 262-269 (1991).

31) Haghgoo S., Hasegawa T., Nadai M., Wang L., Ishigaki T., Miyamoto K., Nabeshima T., J. Pharm. Pharmacol., 47, 412- 419 (1995).

32) Omura T., Sato R., J. Biol. Chem., 239, 2370-2378 (1964).

33) Burke M. D., Thompson S., Weaver R. J., Wolf C. R., Mayer R. T., Biochem. Pharmacol., 48, 923-936 (1994).

34) Kitaichi K., Nakayama H., Ueyama J., Nadai M., Baba K., Takagi Kenj., Takagi Kenz., Ohta M., Hasegawa T., Biochem. Pharmacol., 67, 1427-1435 (2004).

35) Miyoshi M., Nadai M., Nitta A., Ueyama J., Shimizu A., Takagi Kenj., Nabeshima T., Takagi Kenz., Saito K., Hasegawa T., Eur. J. Pharmacol., 507, 229-237 (2005).

36) Ueyama J., Nadai M., Kanazawa H., Iwase M., Nakayama H., Hashimoto K., Yokoi T., Baba K., Takagi Kenj., Takagi Kenz., Hasegawa T., Eur. J. Pharmacol., 510, 127-134 (2005).

37) Wang L., Hasegawa T., Nadai M., Muraoka I., Nabeshima T., Kato N., J. Pharm. Pharmacol., 45, 34-38 (1993).

38) Dische Z., Borenfreund E., J. Biol. Chem., 192, 583-587 (1951).

39) Butler M. A., Iwasaki M., Guengerich F. P., Kadlubar F. F., Proc. Natl. Acad. Sci. U.S.A., 86, 7696-7700 (1989).

40) Miners J. O., Birkett D. J., Gen. Pharmacol., 27, 245-249 (1996).

41) Tassaneeyakul W., Birkett D. J., Veronese M. E., McManus M. E., Tukey R. H., Quattrochi L. C., Gelboin H. V., Miners J. O., J. Pharmacol. Exp. Ther, 265, 401- 407 (1993).

42) Prior T. I., Chue P. S., Tibbo P., Baker G. B., Eur. Neuropsychophar- 
macol., 9, 301-309 (1999).

43) Yoshimoto K., Echizen H., Chiba K., Tani M., Ishizaki T., Br. J. Clin. Pharmacol., 39, 421-431 (1995).

44) Steingrimsdottir H., Gruber A., Palm C., Grimfors G., Kalin M., Eksborg S., Antimicrob. Agents Chemother., 44, 207-209 (2000).

45) Fujioka Y., Mizuno N., Morita E., Motozono H., Takahashi K., Yamanaka Y., Shinkuma D., J. Pharm. Pharmacol., 43, 463-469 (1991).
46) Burgess E. D., Gill M. J., J. Clin. Pharmacol., 30, 997-1000 (1990).

47) Eagling V. A., Tjia J. F., Back D. J., Br. J. Clin. Pharmacol., 45, 107114 (1998).

48) Kobayashi K., Urashima K., Shimada N., Chiba K., Drug Metab. Dispos., 31, 833-836 (2003).

49) Kuh H. J., Shim C. K., Arch. Pharmacol. Res., 17, 124-130 (1994). 